Article

\title{
Enhancement of Intracavity-Pumped Terahertz Parametric Oscillator Power by Adopting Diode-Side Pumped Configuration Based on $\mathrm{KTiOPO}_{4}$ Crystal
}

\author{
Feilong Gao ${ }^{D}$, Xingyu Zhang *, Zhenhua Cong, Zhaojun Liu, Xiaohan Chen, Zengguang Qin, \\ Peng Wang, Jinjin $\mathrm{Xu}$, Zecheng Wang and Na Ming
}

School of Information Science and Engineering, Shandong Provincial Key Laboratory of Laser Technology and Application, Shandong University, Qingdao 266237, China; 201520237@mail.sdu.edu.cn (F.G.); congzhenhua@sdu.edu.cn (Z.C.); zhaojunliu@sdu.edu.cn (Z.L.); cxh@sdu.edu.cn (X.C.); qinzengguang@sdu.edu.cn (Z.Q.); 201712312@mail.sdu.edu.cn (P.W.); 201732375@mail.sdu.edu.cn (J.X.); 201920408@mail.sdu.edu.cn (Z.W.); 201920404@mail.sdu.edu.cn (N.M.)

* Correspondence: xyz@sdu.edu.cn

Received: 20 November 2019; Accepted: 9 December 2019; Published: 11 December 2019

\begin{abstract}
In this paper, a $\mathrm{KTiOPO}_{4}(\mathrm{KTP})$ crystal is used as the nonlinear medium in an intracavity-pumped terahertz parametric oscillator (TPO) based on stimulated polariton scattering (SPS). Almost all the reported intracavity SPS sources adopt the diode-end pumped configuration, and the THz output power is limited by the serious thermal effect and small fundamental beam size. For improving the $\mathrm{THz}$ output power, we propose diode-side pumping for the laser medium to get a higher fundamental power and a larger fundamental beam size. A convex-plane fundamental laser resonator is used to further offset the thermal effect and increase the fundamental beam size. The $\mathrm{THz}$ frequency of the intracavity-pumped KTP terahertz parametric oscillator can be discontinuously tuned from $3.19 \mathrm{THz}$ to $5.94 \mathrm{THz}$ with three gaps. The fundamental beam diameter in the KTP crystal is about $1.3 \mathrm{~mm}$. The maximum average THz power is $166 \mu \mathrm{W}$ at $5.74 \mathrm{THz}$ under a pulse repetition frequency (PRF) of $6 \mathrm{kHz}$ and a diode pump power of $98 \mathrm{~W}$. By means of the diode-side pumped configuration, the maximum $\mathrm{THz}$ output power is more than two-fold higher compared to the diode-end pumped configuration reported using the KTP crystal.
\end{abstract}

Keywords: $\mathrm{KTiOPO}_{4}$ crystal; terahertz parametric oscillator; nonlinear optics; diode-side pumping

\section{Introduction}

$\mathrm{KiTiOPO}_{4}(\mathrm{KTP})$ is an outstanding optical nonlinear medium. It has many excellent properties such as a large nonlinear coefficient $\left(d_{33}=15.4 \mathrm{pm} / \mathrm{V}\right)$ [1], high optical damage threshold $\left(10 \mathrm{~J} / \mathrm{cm}^{2}\right.$ at $10 \mathrm{~Hz}, 6 \mathrm{~ns}, 1064 \mathrm{~nm}$ ) [2], high transparency range, etc. The KTP crystal is used as a nonlinear medium to extend the wavelength range of a practical laser source. It has good performance in second-order nonlinear effects such as second harmonic generation (SHG) $[3,4]$, sum frequency generation (SFG) $[5,6]$, and optical parametric oscillation (OPO) [7,8], and third-order nonlinear effects such as stimulated Raman scattering (SRS) $[9,10]$.

In recent years, researchers found that the KTP crystal could also be used to obtain $\mathrm{THz}$ radiation through the stimulated polariton scattering (SPS) effect [11-14]. SPS is a special frequency down-converting process which includes both second-order and third-order nonlinear effects [15]. When a strong pump wave (usually in the near-infrared range) is incident into the polar crystal which is both infrared-active and Raman-active, a near-infrared wave called a Stokes wave and a far-infrared wave with $\mathrm{THz}$ frequency are parametrically generated according to the energy conservation law and 
the momentum conservation law. Because the refractive index of the nonlinear crystal in the $\mathrm{THz}$ wave spectral range is large, the SPS process must obey the non-collinear phase matching condition.

In 2014, Wang reported an external cavity-pumped SPS source using a KTP crystal. This is the first time that a nonlinear medium different from the traditional crystal $\mathrm{LiNbO}_{3}(\mathrm{LN})$ was realized to generate $\mathrm{THz}$ radiation based on SPS. Different from the $\mathrm{THz}$ frequency of $0.6-3.5 \mathrm{THz}$ obtained from the LN crystal [16-20], the generated THz frequency could be tuned from $3.17 \mathrm{THz}$ to $6.13 \mathrm{THz}$ by using the KTP crystal. The obtained THz pulse energy was $336 \mathrm{~nJ}$ at $5.72 \mathrm{THz}$ when the pulse repetition frequency (PRF) was $10 \mathrm{~Hz}$ and the pump pulse energy was $80 \mathrm{~mJ}$ [11]. In the following years, there were some reports of enhancing the performance of the external cavity-pumped SPS laser using a KTP crystal [12-14]. The external cavity-pumped SPS sources are mainly used to generate terahertz pulses with relatively high pulse energy and low pulse repetition rate.

Compared with the external cavity-pumped system which requires a high-pulse-energy pumping laser, an intracavity-pumped system, in which the nonlinear crystal is inserted in the fundamental cavity, can take advantage of the high intracavity fundamental power density to enhance nonlinear conversion. Intracavity-pumped SPS sources are usually compact and exhibit relatively low SPS thresholds, high pulse repetition rates, high average $\mathrm{THz}$ output powers, and high diode-to- $\mathrm{THz}$ conversion efficiencies. The KTP crystal is also used as the nonlinear medium in intracavity-pumped SPS sources. Ortega reported an intracavity-pumped SPS laser using a KTP crystal in his PhD thesis. The diode-end pumped configuration for fundamental gain medium was adopted. The generated average $\mathrm{THz}$ power was $70.9 \mu \mathrm{W}$ at $5.58 \mathrm{THz}$ for a diode power of $7.3 \mathrm{~W}$ [21].

This diode-end pumped configuration is used in nearly all intracavity SPS sources [22-27]. Although the laser threshold is low and the diode-to-THz conversion efficiency is high in the diode-end pumped configuration because of the high overlapping efficiency between the diode wave and the fundamental wave, further improving the THz output power is difficult for several reasons. Firstly, the generations of the Stokes and $\mathrm{THz}$ waves arise from the consumption of the fundamental wave. In the diode-end pumped configuration, the diode power and the intracavity fundamental power are limited by the thermal fracture of the laser material [28]. Secondly, the SPS process must obey the non-collinear phase matching condition. The interaction volume among the fundamental, Stokes, and $\mathrm{THz}$ beams is positively correlated with the fundamental beam size and the overlap of the non-collinear Stokes beam (see Figure 1). However, the fundamental diameter in the diode-end pumped configuration is usually as small as several hundred micrometers. Diode-side pumping for the laser medium is an outstanding method to obtain high output power. It is used in the fields of single-mode laser [29,30], second harmonic generation [31,32], and stimulated Raman scattering [33,34]. Because the diode laser is uniformly incident into the laser rod, the thermal effect is relatively light in the diode-side pumped configuration. Thus, the system can operate efficiently even under high diode power [35]. In addition, the fundamental beam size is larger compared with that in the diode-end pumped configuration [36]. Of course, it should be pointed out that the diode-side pumped systems have the common disadvantage that the optical-to-optical efficiency is lower due to the insufficient absorption of the diode pump light in the outer part of the laser rod.

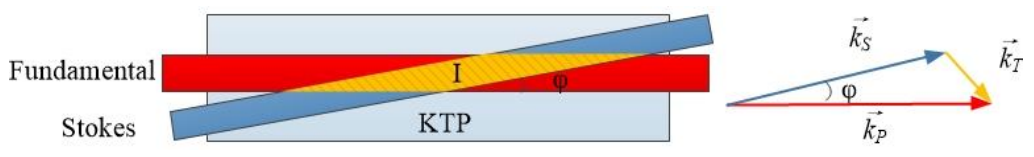

Figure 1. The spatial interaction between the fundamental and Stokes waves, and the non-collinear phase-matching condition.

In this paper, a KTP crystal was used as the nonlinear medium in an intracavity SPS source. The fundamental beam size and fundamental power were increased by means of side pumping for the laser medium to enhance the THz output power. The fundamental cavity was designed as a plane-convex configuration to further increase the fundamental beam size and offset some of the thermal 
effect. Finally, the fundamental beam diameter in the KTP crystal was about $1.3 \mathrm{~mm}$. The terahertz wave frequency could be intermittently tuned from 3.19 to $5.94 \mathrm{THz}$ with three gaps. The obtained maximum $\mathrm{THz}$ average power was $166 \mu \mathrm{W}$ at $5.74 \mathrm{THz}$ under a diode power of $98 \mathrm{~W}$ and a PRF of $6 \mathrm{kHz}$. The maximum THz output power was more than two-fold higher compared to that obtained in the intracavity-pumped SPS laser using a KTP crystal with diode-end pumped configuration.

\section{Experimental Set-Up}

Figure 2 depicts the experimental schematic of the intracavity-pumped KTP terahertz parametric oscillator (TPO). Diode-side pumping for the gain medium of neodymium-doped yttrium aluminum garnet (Nd:YAG) was adopted. The diameter and length of the Nd:YAG rod (doping concentration of 1 at.\%; anti-reflection coated at $1064 \mathrm{~nm}$ ) were $3 \mathrm{~mm}$ and $65 \mathrm{~mm}$, respectively. The maximum power of the diode laser was $180 \mathrm{~W}$. The fundamental laser was Q-switched by an acousto-optic module (Gooch and Housego Company, Ilminster, Somerset, United Kingdom) which was coated with an anti-reflection (AR) film at $1064 \mathrm{~nm}$. The fundamental resonator was composed of M1, M2, and the total reflection surface of the KTP crystal. Compared with the reported intracavity-pumped terahertz parametric sources in which the fundamental resonators were plane-plane or plane-concave configurations, the fundamental cavity was a convex-plane configuration. This could offset the thermal effect to some degree and further increase the fundamental beam size. The total fundamental cavity length was $320 \mathrm{~mm}$. Mirror M1 was convex with a curvature radius of $2000 \mathrm{~mm}$. It was coated with a high-reflection (HR) film at $1064 \mathrm{~nm}(R>99.9 \%)$. The output mirror M2 was flat with a reflectivity of $99.54 \%$ at $1064 \mathrm{~nm}$. The Stokes cavity consisted of two plane mirrors (M3 and M4), and the cavity length was $100 \mathrm{~mm}$. In order to avoid clipping the fundamental beam, M3 and M4 were processed as the same semicircle shape. M3 was HR coated from $1060 \mathrm{~nm}$ to $1100 \mathrm{~nm}(R>99.9 \%)$, and M4 was partial transmission coated from $1060 \mathrm{~nm}$ to $1100 \mathrm{~nm}(T=20 \%$ at $1086 \mathrm{~nm})$. The KTP crystal had an isosceles trapezoid shape in the $x y$ plane, and the base angle was designed to be about $60^{\circ}$. The two waist planes of the KTP crystal were the optical windows for the fundamental and Stokes waves, and they were AR coated from $1060 \mathrm{~nm}$ to $1100 \mathrm{~nm}$. This special shape allowed the $\mathrm{THz}$ wave to be radiated nearly perpendicularly through the base plane of the KTP crystal with no need for any couplers, and it allowed the fundamental and Stokes waves to be totally reflected on the $\mathrm{THz}$ output surface. The waist and base lines of the isosceles trapezoid were $15 \mathrm{~mm}$ and $26 \mathrm{~mm}$, respectively. The thickness of the KTP crystal was $7 \mathrm{~mm}$. The Stokes cavity mirrors were installed on two independent electromechanical rotating platforms. The tuning characteristics of the Stokes and $\mathrm{THz}$ waves could be realized by precisely controlling the axis of the Stokes cavity. The THz signal was received by a calibrated Golay cell (Tydex, GC-1D, Saint Petersburg, Leningrad Region, Russia). In order to avoid the interference of the undesired signals, a long-pass filter (TYDEX, LPF14.3-47, Saint Petersburg, Leningrad Region, Russia) was installed in front of the Golay cell. The duty cycle and operation frequency of the chopper between the $\mathrm{THz}$ output surface and the Golay cell were $50 \%$ and $10 \mathrm{~Hz}$, respectively.

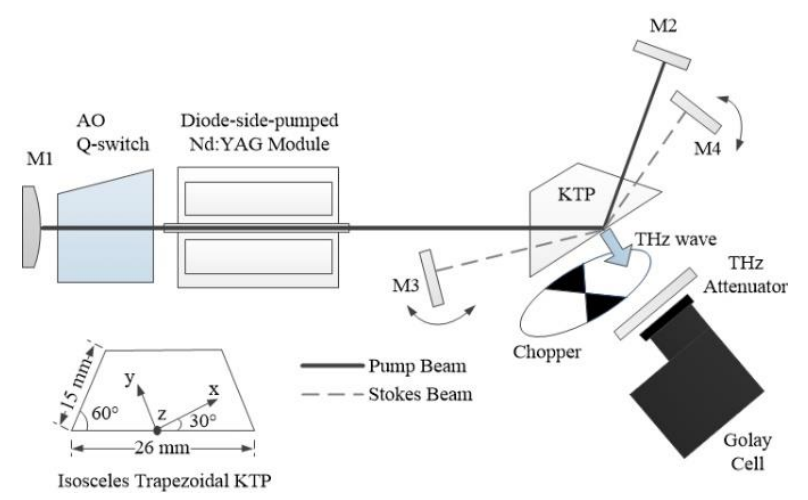

Figure 2. Experimental set-up of the intracavity-pumped terahertz parametric oscillator (TPO) using a $\mathrm{KTiOPO}_{4}(\mathrm{KTP})$ crystal with diode-side pumped configuration. 


\section{Results and Discussion}

The tuning characteristics of the intracavity KTP TPO with diode-side pumped configuration were investigated under a diode power of $80 \mathrm{~W}$ and a PRF of $6 \mathrm{kHz}$, as shown in Figure 3. A spectrometer (YOKOGAWA, AQ6315, 350-1750 nm, Musashino-shi, Tokyo, Japan) was used to monitor the Stokes wavelength. The generated Stokes wavelength was discretely tuned from 1076.3 to $1077.4 \mathrm{~nm}$, from 1080.3 to $1081.6 \mathrm{~nm}$, from 1082.6 to $1083.2 \mathrm{~nm}$, and from 1085.4 to $1087.1 \mathrm{~nm}$. The $\mathrm{THz}$ frequency was calculated using the energy conservation law and was tuned from 3.19 to $3.45 \mathrm{THz}$, from 4.21 to $4.53 \mathrm{THz}$, from 4.80 to $4.94 \mathrm{THz}$, and from 5.51 to $5.94 \mathrm{THz}$, respectively. There existed three gaps corresponding to the three $\mathrm{A}_{1}$ transverse modes of the KTP crystal at 134, 154, and $179 \mathrm{~cm}^{-1}$. The absorption for the electromagnetic wave is very large when the frequency is close to those variation modes. The optimal $\mathrm{THz}$ frequency was at $5.74 \mathrm{THz}$ where the $\mathrm{THz}$ output power was maximal.

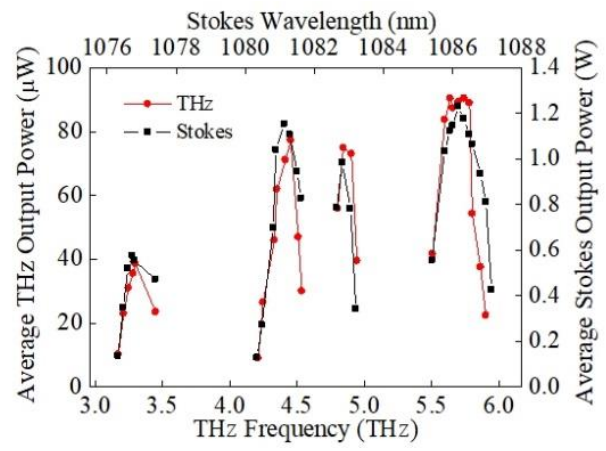

Figure 3. Tuning characteristics of the intracavity diode-side pumped KTP stimulated polariton scattering (SPS) laser.

The shaded area I in Figure 1 depicts the interaction area between the fundamental and Stokes beams in the crystal. The diagram is sketched for linear geometry for simplicity. The interaction area is the same as for a surface-emitted configuration because of the mirror reflection. The fundamental wave was perpendicularly incident into the crystal. The Stokes wave oscillated at an angle $\varphi$ with the fundamental beam according to the near-forward scattering configuration $X(Z Z) X+\varphi$ in the SPS process [37]. The THz wave was generated in the overlapping area I. Obviously, as the fundamental beam size increases in size, so does the overlapping volume among the fundamental, Stokes, and THz waves in the crystal. In particular, for the KTP crystal, the optimal phase-matching angle between the fundamental and Stokes beams ( $2.4^{\circ}$ in the crystal) is larger than that in LN and $\mathrm{RbTiOPO}_{4}$. Thus, the fundamental beam size is more critical to the performance of the KTP SPS laser. The fundamental beam size outside the resonator was measured using a camera beam profiler (THORLABS, BC106N-VIS/M, 350-1100 nm, Newton, New Jersey, United States). The beam radii at different positions were fitted into the curve $\omega^{2}(x)=\omega_{0}^{2}\left[1+(x / f)^{2}\right]$, where $x$ represents the distance between the camera beam profiler and the output coupler M2, $\omega_{0}$ is the beam waist radius, $f=\pi \omega_{0}^{2} /\left(\lambda M^{2}\right)$ is called the Rayleigh length, $M^{2}$ is the beam quality parameter, and $\lambda$ is the wavelength. After curve fitting, we could obtain $\omega_{0}$. Through the ABCD transformation, the fundamental beam diameter inside the KTP crystal could be calculated. The fundamental beam size decreased slightly with increasing diode power. When the diode power was increased from $60 \mathrm{~W}$ to $95 \mathrm{~W}$, the fundamental beam size varied from $1.34 \mathrm{~mm}$ to $1.25 \mathrm{~mm}$. The average $\mathrm{M}^{2}$ values of the fundamental wave in the horizontal and vertical directions were measured at about 2.31 and 2.85, respectively, using an $\mathrm{M}^{2}$ beam quality analysis system (THORLABS, M2MS, 400-5000 nm, Newton, New Jersey, United States).

The average output powers of the fundamental (before and after depletion), Stokes, and $\mathrm{THz}$ waves versus the diode power are shown in Figure 4 when the $\mathrm{THz}$ wave was at the optimal frequency of $5.74 \mathrm{THz}$, and the PRF was $6 \mathrm{kHz}$. By adjusting mirror M4, the SPS process could be switched on 
and off to control the consumption of the fundamental wave and the generation of the Stokes and $\mathrm{THz}$ waves. When the diode pump power was $64 \mathrm{~W}$, the fundamental wave began to be consumed, and the Stokes and $\mathrm{THz}$ waves were generated simultaneously. With increasing diode power, the residual fundamental power was nearly invariant at a level of about $600 \mathrm{~mW}$, but the fundamental consumption rate (the fundamental consumption power divided by the original fundamental power) increased. When the diode pump power increased to $98 \mathrm{~W}$, the fundamental output powers before and after depletion were measured at $1.33 \mathrm{~W}$ and $650 \mathrm{~mW}$ respectively, and the corresponding fundamental wave consumption increased to $51 \%$. For improving the intracavity fundamental power, the transmission of the fundamental output mirror M2 at $1064 \mathrm{~nm}$ was designed to be very low $(\mathrm{T}=0.46 \%)$. The one-way fundamental powers in the cavity before and after consumption were calculated as $289 \mathrm{~W}$ and $141 \mathrm{~W}$, respectively. The average $\mathrm{THz}$ and Stokes output powers increased nearly linearly with increasing diode power. The average $\mathrm{THz}$ output power increased to $166 \mu \mathrm{W}$ for a diode power of $98 \mathrm{~W}$. A higher diode power was not tried in consideration of the optical damage threshold of the KTP crystal. The diode-to-THz conversion efficiency in this paper is relatively low compared with that in the reported diode-end pumped configuration based on the KTP crystal. However, the maximum average $\mathrm{THz}$ output power was more than doubled $(166 \mu \mathrm{W}$ versus $70.9 \mu \mathrm{W})$.

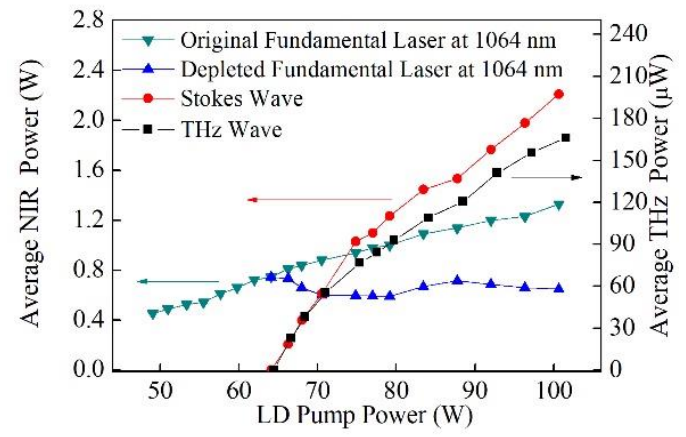

Figure 4. Input-output characteristics of the intracavity KTP SPS laser with diode-side pumped configuration.

The beam patterns of the original fundamental, depleted fundamental, and Stokes waves are shown in Figure 5 when the diode power was 98 W. Because of the diode-side pumped configuration, the beam quality of the fundamental wave was not very good. The mode structure of the fundamental wave became a little distorted after depletion. In contrast, the beam quality of the Stokes wave was much better. Furthermore, there was no obvious change in the Stokes mode profile with increasing diode power. This phenomenon was similar to the beam clean-up effect [38] that occurs in general Raman lasers in which the Raman radiation almost oscillates as a single transverse mode even when the fundament wave oscillates on several transverse modes. The horizontal and vertical beam quality parameters of the Stokes wave were measured as 1.19 and 1.20, respectively.

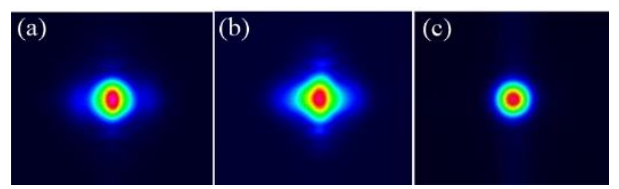

Figure 5. Mode profiles of the (a) original fundamental, (b) depleted fundamental, and (c) Stokes waves.

The pulse waveforms of the fundamental pulse (before and after depletion) and the Stokes pulse were detected by two Si detectors (THORLABS, DET10A/M, 400-1100 nm, Newton, New Jersey, United States). An oscilloscope (Tektronix, TDS5052B, 500MHz, 5GS/s, Bracknell, Berkshire, United Kingdom) was used to record the pulse temporal behaviors. The SPS threshold was $64 \mathrm{~W}$. Figure 6a shows the temporal characteristics of the original fundamental, depleted fundamental, and Stokes pulses under a 
diode power of $70 \mathrm{~W}$. The fundamental pulse was depleted at the falling edge. The Stokes pulse rose at the point where the fundamental wave depletion commenced. The build-up time of the Stokes pulse was about 82 ns. The fundamental pulse widths (all pulse widths were full width half maximum) before and after depletion were 131 ns and 91 ns, respectively. The Stokes pulse width was 22 ns. With increasing diode power, the fundamental depletion was stronger. The pulse widths of the depleted fundamental and Stokes waves decreased. The time interval between the fundamental and Stokes pulses also decreased because the Stokes pulse needed less time to accumulate in the Stokes cavity under higher pump power. Figure $6 \mathrm{~b}$ shows the temporal characteristics of the original fundamental, depleted fundamental, and Stokes pulses under a diode power of $98 \mathrm{~W}$. The fundamental pulse was significantly consumed just after it passed through the peak. This corresponds to the optimum condition in which the majority of the stored accumulated population inversion in the gain medium Nd:YAG was extracted through Q-switching into the circulating fundamental field. The fundamental pulse widths before and after depletion were $110 \mathrm{~ns}$ and $46 \mathrm{~ns}$, respectively. The Stokes pulse width was 12 ns. The time interval between the fundamental and Stokes pulses was about 73 ns.
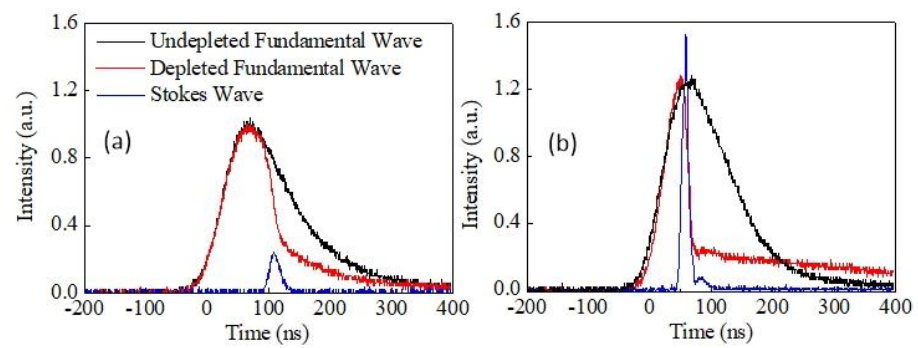

Figure 6. Waveforms of the fundamental (before and after depletion) and Stokes pulses under diode powers of (a) $70 \mathrm{~W}$ and (b) $98 \mathrm{~W}$.

\section{Conclusions}

A KTP crystal was used as the nonlinear medium in an intracavity SPS source. With a desire to improve the average $\mathrm{THz}$ output power, the diode-side pumped configuration was employed to obtain a high intracavity fundamental power and a large fundamental beam size. The fundamental cavity was designed as a convex-plane configuration to offset some of the thermal effect and further increase the interaction volume among the fundamental, Stokes, and $\mathrm{THz}$ waves. The THz wave was efficiently emitted from the KTP crystal by adopting a surface-emitted configuration. The generated $\mathrm{THz}$ frequency could be tuned from $3.19 \mathrm{THz}$ to $5.94 \mathrm{THz}$ with three gaps. The optimal $\mathrm{THz}$ frequency was $5.74 \mathrm{THz}$ where the average $\mathrm{THz}$ power was maximal. The fundamental beam diameter in the KTP crystal was about $1.3 \mathrm{~mm}$. Finally, the maximum THz output power of $166 \mu \mathrm{W}$ at $5.74 \mathrm{THz}$ was obtained under a diode power of $98 \mathrm{~W}$, and the fundamental wave depletion increased to $51 \%$. Compared with the diode-end pumped configuration reported using the KTP crystal, the maximum average $\mathrm{THz}$ output power was more than doubled.

Author Contributions: Conceptualization, F.G. and X.Z.; methodology, F.G. and X.Z.; investigation, F.G.; resources, P.W., J.X., and Z.W.; writing-original draft preparation, F.G.; writing-review and editing, Z.C., Z.L., and X.C.; visualization, Z.Q. and N.M.

Funding: This research was funded by the National Natural Science Foundation of China, grant numbers 61775122, 61475087, 61605103; the Key Technology Research and Development Program of Shandong, grant numbers 2017CXGC0809, 2017CXGC0809; and the Natural Science Foundation of Shandong Province, grant number ZR2014FM024.

Acknowledgments: The calibration work of the Golay cell was supported by the Institute of Semiconductors, CAS.

Conflicts of Interest: The authors declare no conflicts of interest. 


\section{References}

1. Shoji, I.; Kondo, T.; Shirane, A.M.; Ito, R. Absolute scale of second-order nonlinear-optical coefficients. J. Opt. Soc. Am. 1997, 14, 2268-2294. [CrossRef]

2. Hildenbrand, A.; Wagner, F.R.; Akhouayri, H.; Natoli, J.Y.; Commandre, M.; Théodore, F.; Albrecht, H. Laser-induced damage investigation at $1064 \mathrm{~nm}$ in $\mathrm{KTiOPO}_{4}$ crystals and its analogy with $\mathrm{RbTiOPO}_{4}$. Appl. Opt. 2009, 48, 4263-4269. [CrossRef] [PubMed]

3. Driscoll, T.A.; Hoffman, H.J.; Stone, R.E.; Perkins, P.E. Efficient second-harmonic generation in KTP crystals. J. Opt. Soc. Am. B 1986, 3, 683-686. [CrossRef]

4. Brown, A.J.; Bowers, M.S.; Kangas, K.W.; Fisher, C.H. High-energy, high-efficiency second-harmonic generation of 1064-nm radiation in KTP. Opt. Lett. 1992, 17, 109-111. [CrossRef] [PubMed]

5. Stolzenberger, R.A.; Hsu, C.C.; Peyghambarian, N.; Reid, J.J.; Morgan, R.A. Type II sum frequency generation in flux and hydrothermally grown KTP at 1.319 and $1.338 \mu \mathrm{m}$. IEEE Photon. Technol. Lett. 1989, 1, 446-448. [CrossRef]

6. Chen, Y.F.; Chen, Y.S.; Tsai, S.W. Diode-pumped Q-switched laser with intracavity sum frequency mixing in periodically poled KTP. Appl. Phys. B 2004, 79, 207-210. [CrossRef]

7. Kato, K. Parametric oscillation at $3.2 \mu \mathrm{m}$ in KTP pumped at $1.064 \mu \mathrm{m}$. IEEE J. Quantum Electron. 1991, 27, 1137-1140. [CrossRef]

8. Lin, J.T.; Montgomery, J.L. Generation of tunable mid-IR (1.8-2.4 $\mu \mathrm{m})$ laser from optical parametric oscillation in KTP. Opt. Commun. 1990, 75, 315-320. [CrossRef]

9. Su, F.F.; Zhang, X.Y.; Wang, W.T.; Cong, Z.H.; Shi, M.; Yang, X.Q.; Kong, W.J.; Ma, L.L.; Wu, W.D. High-efficient diode-pumped actively Q-switched Nd: YAG/KTP Raman laser at $1096 \mathrm{~nm}$ wavelength. Opt. Commun. 2013, 305, 201-203. [CrossRef]

10. Lee, C.Y.; Chang, C.C.; Sung, C.L.; Chen, Y.F. Intracavity continuous-wave multiple stimulated-Raman-scattering emissions in a KTP crystal pumped by a $\mathrm{Nd}: \mathrm{YVO}_{4}$ laser. Opt. Express 2015, 23, 22765-22770. [CrossRef]

11. Wang, W.T.; Cong, Z.H.; Chen, X.H.; Zhang, X.Y.; Qin, Z.G.; Tang, G.Q.; Li, N.; Wang, C.; Lu, Q.M. Terahertz parametric oscillator based on $\mathrm{KTiOPO}_{4}$ crystal. Opt. Lett. 2014, 39, 3706-3709. [CrossRef] [PubMed]

12. Yan, C.; Wang, Y.Y.; Xu, D.G.; Xu, W.T.; Liu, P.X.; Yan, D.X.; Duan, P.; Zhong, K.; Shi, W.; Yao, J.Q. Green laser induced terahertz tuning range expanding in $\mathrm{KTiOPO}_{4}$ terahertz parametric oscillator. Appl. Phys. Lett. 2016, 108, 011107. [CrossRef]

13. Wu, M.H.; Chiu, Y.C.; Wang, T.D.; Zhao, G.; Zukauskas, A.; Laurell, F.; Huang, Y.C. Terahertz parametric generation and amplification from potassium titanyl phosphate in comparison with lithium niobate and lithium tantalite. Opt. Express 2016, 24, 25964-25973. [CrossRef] [PubMed]

14. Wang, Y.Y.; Ren, Y.C.; Xu, D.G.; Tang, L.H.; He, Y.X.; Song, C.; Chen, L.Y.; Li, C.Z.; Yan, C.; Yao, J.Q. Energy scaling and extended tunability of a ring cavity terahertz parametric oscillator based on $\mathrm{KTiOPO}_{4}$ crystal. Chin. Phys. B 2018, 27, 114213. [CrossRef]

15. Kawase, K.; Shikata, J.I.; Ito, H. Terahertz wave parametric source. Phys. D Appl. Phys. 2002, 35, R1. [CrossRef]

16. Shikata, J.I.; Kawase, K.; Karino, K.I.; Taniuchi, T.; Ito, H. Tunable terahertz-wave parametric oscillators using $\mathrm{LiNbO}_{3}$ and $\mathrm{MgO}: \mathrm{LiNbO}_{3}$ crystals. IEEE Trans. Microw. Theory Tech. 2000, 48, 653-661. [CrossRef]

17. Lee, A.; He, Y.; Pask, H. Frequency-Tunable THz Source Based on Stimulated Polariton Scattering in $\mathrm{Mg}: \mathrm{LiNbO}_{3}$. IEEE J. Quantum Electron. 2013, 49, 357-364. [CrossRef]

18. Wang, W.T.; Zhang, X.Y.; Wang, Q.P.; Cong, Z.H.; Chen, X.H.; Liu, Z.J.; Wang, C. Multiple-beam output of a surface-emitted terahertz-wave parametric oscillator by using a slab $\mathrm{MgO}: \mathrm{LiNbO}_{3}$ crystal. Opt. Lett. 2014, 39, 754-757. [CrossRef]

19. Tang, G.Q.; Cong, Z.H.; Qin, Z.G.; Zhang, X.Y.; Wang, W.T.; Wu, D.; Zhang, S.J. Energy scaling of terahertz-wave parametric sources. Opt. Express 2015, 23, 4144-4152. [CrossRef]

20. Zhang, R.L.; Qu, Y.C.; Zhao, W.J.; Liu, C.; Chen, Z.L. Si-prism-array coupled terahertz-wave parametric oscillator with pump light totally reflected at the terahertz-wave exit surface. Opt. Lett. 2016, 41, 4016-4019. [CrossRef]

21. Ortega, T.A. Frequency Extension of Solid-State Terahertz Lasers. Ph.D. Thesis, Macquarie University, Sydney, Australia, 2017. 
22. Edwards, T.J.; Walsh, D.; Spurr, M.B.; Rae, C.F.; Dunn, M.H.; Browne, P.G. Compact source of continuously and widely-tunable terahertz radiation. Opt. Express 2006, 14, 1582-1589. [CrossRef] [PubMed]

23. Walsh, D.A.; Browne, P.G.; Dunn, M.H.; Rae, C.F. Intracavity parametric generation of nanosecond terahertz radiation using quasi-phase-matching. Opt. Express 2010, 18, 13951-13963. [CrossRef] [PubMed]

24. Lee, A.J.; Pask, H.M. Continuous wave, frequency-tunable terahertz laser radiation generated via stimulated polariton scattering. Opt. Lett. 2014, 39, 442-445. [CrossRef] [PubMed]

25. Ortega, T.A.; Pask, H.M.; Spence, D.J.; Lee, A.J. Stimulated polariton scattering in an intracavity RbTiOPO4 crystal generating frequency-tunable THz output. Opt. Express 2016, 24, 10254-10264. [CrossRef] [PubMed]

26. Ortega, T.A.; Pask, H.M.; Spence, D.J.; Lee, A.J. THz polariton laser using an intracavity Mg: $\mathrm{LiNbO}_{3}$ crystal with protective teflon coating. Opt. Express 2017, 25, 3991-3999. [CrossRef] [PubMed]

27. Lee, A.J.; Spence, D.J.; Pask, H.M. Tunable THz polariton laser based on $1342 \mathrm{~nm}$ wavelength for enhanced terahertz wave extraction. Opt. Lett. 2017, 42, 2691-2694. [CrossRef] [PubMed]

28. Tidwell, S.C.; Seamans, J.F.; Bowers, M.S.; Cousins, A.K. Scaling CW diode-end-pumped Nd:YAG lasers to high average powers. IEEE J. Quantum Electron. 1992, 28, 997-1009. [CrossRef]

29. Welford, D.; Rines, D.M.; Dinerman, B.J. Efficient $\mathrm{TEM}_{00}$-mode operation of a laser-diode side-pumped Nd: YAG laser. Opt. Lett. 1991, 16, 1850-1852. [CrossRef]

30. Konno, S.; Fujikawa, S.; Yasui, K. 80W cw TEM $001064 \mathrm{~nm}$ beam generation by use of a diode-side-pumped Nd:YAG rod laser. Appl. Phys. Lett. 1997, 70, 2650-2651. [CrossRef]

31. Yi, J.; Moon, H.J.; Lee, J. Diode-pumped 100-W green Nd:YAG rod laser. Appl. Opt. 2004, 43, $3732-3737$. [CrossRef]

32. Xu, D.; Wang, Y.; Li, H.; Yao, J.; Tsang, Y.H. $104 \mathrm{~W}$ high stability green laser generation by using diode laser pumped intracavity frequency-doubling Q-switched composite ceramic Nd:YAG laser. Opt. Express 2007, 15, 3991-3997. [CrossRef] [PubMed]

33. Li, S.T.; Zhang, X.Y.; Wang, Q.P.; Zhang, X.L.; Cong, Z.H.; Zhang, H.N.; Wang, J.Y. Diode-side-pumped intracavity frequency-doubled $\mathrm{Nd}$ : $\mathrm{YAG} / \mathrm{BaWO}_{4}$ Raman laser generating average output pow of $3.14 \mathrm{~W}$ at 590 nm. Opt. Lett. 2007, 32, 2951-2953. [CrossRef] [PubMed]

34. Li, C.Y.; Bo, Y.; Yang, F.; Wang, Z.C.; Xu, Y.T.; Wang, Y.B.; Gao, H.W.; Peng, Q.J.; Cui, D.F.; Xu, Z.Y. 106.5 W high beam quality diode-side-pumped Nd:YAG laser at 1123 nm. Opt. Express 2010, 18, 7923-7928.

35. Kojima, T.; Yasui, K. Efficient diode side-pumping configuration of a Nd:YAG rod laser with a diffusive cavity. Appl. Opt. 1997, 36, 4981-4984. [CrossRef]

36. Golla, D.; Knoke, S.; Schöne, W.; Ernst, G.; Bode, M.; Tünnermann, A.; Welling, H. 300-W cw diode-laser side-pumped Nd:YAG rod laser. Opt. Lett. 1995, 20, 1148-1150. [CrossRef] [PubMed]

37. Wang, W.T.; Cong, Z.H.; Liu, Z.J.; Zhang, X.Y.; Qin, Z.G.; Tang, G.Q.; Li, N.; Zhang, Y.G.; Lu, Q.M. THz-wave generation via stimulated polariton scattering in $\mathrm{KTiOAsO}_{4}$ crystal. Opt. Express 2014, 22, 17092-17098. [CrossRef] [PubMed]

38. Murray, J.T.; Austin, W.L.; Powell, R.C. Intracavity Raman conversion and Raman beam cleanup. Opt. Mater. 1999, 11, 353-371. [CrossRef]

(C) 2019 by the authors. Licensee MDPI, Basel, Switzerland. This article is an open access article distributed under the terms and conditions of the Creative Commons Attribution (CC BY) license (http://creativecommons.org/licenses/by/4.0/). 